\title{
A bridge between the minimal doubly resolving set problem in (folded) hypercubes and the coin weighing problem*
}

\author{
Changhong Lu Qingjie $\mathrm{Ye}^{\dagger}$ \\ School of Mathematical Sciences, \\ Shanghai Key Laboratory of PMMP, \\ East China Normal University, \\ Shanghai 200241, P. R. China \\ Email: chlu@math.ecnu.edu.cn \\ Email: mathqjye@stu.ecnu.edu.cn
}

\begin{abstract}
In this paper, we consider the minimal doubly resolving set problem in Hamming graphs, hypercubes and folded hypercubes. We prove that the minimal doubly resolving set problem in hypercubes is equivalent to the coin weighing problem. Then we answer an open question on the minimal doubly resolving set problem in hypercubes. We disprove a conjecture on the metric dimension problem in folded hypercubes and give some asymptotic results for the metric dimension and the minimal doubly resolving set problems in Hamming graphs and folded hypercubes by establishing connections between these problems. Using the Lindström's method for the coin weighing problem, we give an efficient algorithm for the minimal doubly resolving set problem in hypercubes and report some new upper bounds. We also prove that the minimal doubly resolving set problem is NP-hard even restrict on split graphs, bipartite graphs and co-bipartite graphs.
\end{abstract}

Keywords: Metric dimension, Doubly resolving set, Coin weighing problem, Hypercube, Hamming graph

\section{Introduction}

Let $G$ be a finite, connected, simple and undirected graph with vertex set $V=V(G)$ and edge set $E=E(G)$. The distance between vertices $u$ and $v$ is denoted by $d_{G}(u, v)$. The Cartesian product of graphs $G$ and $H$, denoted by $G \square H$, where $V(G \square H)=\{(g, h): g \in V(G), h \in V(H)\}$, and $\left(g_{1}, h_{1}\right)\left(g_{2}, h_{2}\right) \in E(G \square H)$ if and only if $g_{1}=g_{2}, h_{1} h_{2} \in E(H)$ or $g_{1} g_{2} \in E(G), h_{1}=h_{2}$. The cartesian product is associative and $G_{1} \square G_{2} \square \cdots \square G_{d}$ is well-defined.

The metric dimension problem was independently defined by Slater [32], Harary and Melter [12]. A vertex subset $S$ resolves a graph $G$ if every vertex is uniquely determined by its vector of distances to the vertices in $S$. More formally, a vertex $x$ of $G$ resolves two vertices $u$ and $v$ of $G$ if $d_{G}(u, x) \neq d_{G}(v, x)$. A vertex subset $S$ is a resolving set of $G$ if every two vertices in $G$ are resolved by some vertex of $S$. A resolving set $S$ of $G$ with the minimum cardinality is a metric basis of $G$, and the size of $S$ is the metric dimension of $G$, denoted by $\beta(G)$.

\footnotetext{
*Supported in part by National Natural Science Foundation of China (No. 11871222), Science and Technology Commission of Shanghai Municipality (Nos. 18dz2271000, 19JC1420100) and the Open Research Fund of Key Laboratory of Advanced Theory and Application in Statistics and Data Science-MOE, ECNU.

${ }^{\dagger}$ Corresponding author
} 
Doubly resolving sets were introduced by Cáceres et al. [5] as a tool for researching resolving sets of Cartesian products of graph. Let $G$ be a graph of order $n \geq 2$. We say that $\{x, y\}$ doubly resolves $\{u, v\}$, if $d_{G}(u, x)-d_{G}(u, y) \neq d_{G}(v, x)-d_{G}(v, y)$. A vertex subset $S$ of $G$ is a doubly resolving set of $G$ if every pair of distinct vertices in $G$ is doubly resolved by some pair of vertices in $S$. Let $\Psi(G)$ denote the minimum cardinality of a doubly resolving set of a graph $G \neq K_{1}$. The minimal doubly resolving set problem is determining the minimum cardinality of a doubly resolving set for an input graph $G$.

Let $d_{G}(u, S)=\left(d_{G}\left(u, x_{1}\right), \ldots, d_{G}\left(u, x_{m}\right)\right)$ and $\vec{c}=(c, \ldots, c)$, where $S=\left\{x_{1}, \ldots, x_{m}\right\}$ is a subset of $V(G)$ and $c$ is a constant. Note that the dimension of $\vec{c}$ would be clear from the context. Then for every distinct vertices $u, v \in V(G), S$ is a resolving set if and only if $d(u, S)-d(v, S) \neq \overrightarrow{0}$ while $S$ is a doubly resolving set if and only if $d(u, S)-d(v, S) \neq \vec{c}$ for all constant $c$. Hence $\beta(G) \leq \Psi(G)$. However, there is not a function $f$ such that $\Psi(G) \leq f(\beta(G))$ for all graphs $G$. In fact, Cáceres et al. [5] proved that there is a $k$-connected graph $G_{n, k}$ such that $\beta\left(G_{n, k}\right) \leq 2 k$ and $\Psi\left(G_{n, k}\right) \geq 2 n$ for all $k \geq 1$ and $n \geq 2$. The connection between the two problems is the following theorem that was proved in [5].

Theorem 1.1 (Cáceres et al. [5]). For all graphs $G$ and $H \neq K_{1}$,

$$
\max \{\beta(G), \beta(H)\} \leq \beta(G \square H) \leq \beta(G)+\Psi(H)-1 .
$$

The metric dimension arises in many diverse areas, including network discovery and verification [4], the robot navigation [19] and chemistry [7]. Finding the doubly resolving set in graphs is equivalent to locating the source of a diffusion in complex networks [8]. The metric dimension problem and minimal doubly resolving set problem have many interesting theoretical properties which are out of the scope of this paper. The interested reader is referred, e.g. to [3, 13, 23].

As far as general graphs are concerned, both problems are NP-hard. The proof for the metric dimension problem is given in [19] and for the minimal doubly resolving set problem is given in [24]. Epstein et al. [10] proved that the metric dimension problem is NP-hard even for split graphs, bipartite graphs and co-bipartite graphs. Therefore, some researchers try to design heuristic algorithms to solve the problems. It has been designed the genetic algorithm (GA) to solve the metric dimension problem in [21] and the minimal doubly resolving set problem in [24]. Mladenović et al. [27] designed the variable neighborhood search algorithm (VNS) to solve the metric dimension problem and the minimal doubly resolving set problem. Chartrand et al. [7] and Kratica et al. [24] gave the 0-1 integer linear programming formulations for the metric dimension problem and the minimal doubly resolving set problem respectively.

The Hamming graph $H_{n, q}$ is the Cartesian product of $n$ copies of the complete graph $K_{q}$ with $q$ vertices

$$
H_{n, q}=\underbrace{K_{q} \square K_{q} \square \cdots \square K_{q}}_{n} .
$$

Specifically, the vertex of $H_{n, q}$ is an $n$-dimensional vector $u=\left(u_{1}, \ldots, u_{n}\right) \in\{0,1, \ldots, q-1\}^{n}$ and two vertices are adjacent if they differ in exactly one coordinate (see Figure 1). The operation of addition (subtraction) in $V\left(H_{n, q}\right)$ is defined by the modulo- $q$ addition (subtraction) of the corresponding vector. For example, if $x=(0,0,1,1,2,2)$ and $y=(1,2,2,1,0,2)$ are two vertices of $H_{6,3}$, then $x+y=(1,2,0,2,2,1)$ and $x-y=(2,1,2,0,2,0)$. By the definition of $H_{n, q}$, it is easy to show that $d_{H_{n, q}}(u, v)=d_{H_{n, q}}(u-v, \overrightarrow{0})=$ $\sum_{i=1}^{n} 1_{u_{i} \neq v_{i}}$, where $1_{u_{i} \neq v_{i}}=1$ if $u_{i} \neq v_{i}$ and $1_{u_{i} \neq v_{i}}=0$ if $u_{i}=v_{i}$.

The $n$-dimensional hypercube $Q_{n}$, also called $n$-cube, is a Cartesian product of $n$ copies of $K_{2}$ (see Figure $2)$. Note that $Q_{n}=H_{n, 2}$. For each $u \in V\left(Q_{n}\right)$, we use $\bar{u}$ to denote its opposite vertex, that is $\bar{u}=u+\overrightarrow{1}$. It is clear that $d_{Q_{n}}(u, v)=\sum_{i=1}^{n}\left|u_{i}-v_{i}\right|$ and thus $d_{Q_{n}}(u, \bar{v})=n-d_{Q_{n}}(u, v)$.

The metric dimension of the Hamming graph is connected to Mastermind, which is a deductive game for two players, the code setter and the code breaker. The code setter chooses a secret vector $s=\left(s_{1}, \ldots, s_{n}\right) \in$ $\{0,1, \ldots, q-1\}^{n}$. The task of the code breaker is to infer the secret vector by a series of questions, each a vector $t=\left(t_{1}, \ldots, t_{n}\right) \in\{0,1, \ldots, q-1\}^{n}$. The code setter answers with two integers, denoted by $a(s, t)=\left|\left\{i: s_{i}=t_{i}, 1 \leq i \leq n\right\}\right|$ and $b(s, t)=\max \{a(\tilde{s}, t): \tilde{s}$ is a permutation of $s\}$. The original commercial version of the game is $n=4$ and $q=6$, which was invented by Mordecai Meirowitz. Knuth [20] showed that four questions suffice to determine $s$ in this case. Let $g(n, q)$ be the smallest number such 
that the code breaker can determine any $s$ by asking $g(n, q)$ questions at once (without waiting for the answers). Chvátal [9] proved that $g(n, q) \leq\left(4+2 \log _{q} 2+o(1)\right) n / \log _{q} n$. Kabatianski et al. [16] showed that $\beta\left(H_{n, q}\right)-(q-1) \leq g(n, q) \leq \beta\left(H_{n, q}\right)$. Let $f(n, q)$ be the smallest number such that the code breaker can determine any $s$ by asking $f(n, q)$ questions at once without $b(s, t)$ in the answers. Cáceres et al. [5] showed that $g(n, q) \leq f(n, q)=\beta\left(H_{n, q}\right)$.

It has been showed that $\beta\left(H_{2, q}\right)=\Psi\left(H_{2, q}\right)=\lfloor(4 q-2) / 3\rfloor$ for all $q \geq 5$ by Cáceres et al. [5] and Kratica et al. [22]. Recently, Jiang and Polyanskii [15] gave the following nice theorem.

Theorem 1.2 (Jiang and Polyanskii [15]). $\beta\left(H_{n, q}\right)=(2+o(1)) n / \log _{q} n$ for all $q \geq 2$.

We remark that Kabatyanskiı̌ and Lebedev [17] proved the above theorem for $q=3,4$. For $q=2$, the metric dimension problem in hypercubes is related to the following coin weighing problem.

Given $n$ coins, some of them may be defective. We know the weight $g$ of the good coins in advance and also the weight $h \neq g$ of the defective coins. If we weigh a subset of coins with a spring scale, then the outcome will tell us precisely the number of defective coins among them. The coin weighing problem is determining the minimum number $M(n)$ of weighings by means of which the good and defective coins can be separated under the assumption that all the family of tested subsets has to be given in advance.

More formally, the binary vector $u=\left(u_{1}, \ldots, u_{n}\right) \in\{0,1\}^{n}$ is corresponding to a distribution of defective coins, where $u_{j}=1$ if and only if the $j$-th coin is defective. Similarly, the binary vector $x=\left(x_{1}, \ldots, x_{n}\right) \in$ $\{0,1\}^{n}$ is corresponding to a weighing, where $x_{j}=1$ if and only if the $j$-th coin is chosen to weigh. The outcome of a weighing is a scalar product of $x$ and $u$, that is $u \cdot x=\sum_{i=1}^{n} u_{i} x_{i}$. A set of binary vectors $S$ is called a weighing strategy if for every pair of distinct vectors $u, v$, there exists $x \in S$ such that $u \cdot x \neq v \cdot x$. The coin weighing problem was proposed for $n=5$ by Shapiro [30] and solved by Shapiro and Fine [31]. Erdös and Rényi [11] presented a lower bound and Lindström [25] (independently by Cantor and Mills [6]) presented an upper bound. The lower bound and the upper bound are asymptotically equivalent. Almost all exact values of $M(n)$ are not known yet.

Theorem 1.3 (Cantor and Mills [6], Erdős and Rényi [11], Lindström [25]).

$$
M(n)=(2+o(1)) n / \log _{2} n
$$

A surprising connection between the metric dimension problem in hypercubes and the coin weighing problem was given in [29].

Theorem 1.4 (Sebő and Tannier $[29])$. $\left|\beta\left(Q_{n}\right)-M(n)\right| \leq 1$.

Theorems 1.3 and 1.4 imply that $\beta\left(Q_{n}\right)=(2+o(1)) n / \log _{2} n$. Researchers try to get optimal upper bounds of $\beta\left(Q_{n}\right)$ and $\Psi\left(Q_{n}\right)$ by heuristic algorithms. Besides the genetic algorithm and the variable neighborhood search algorithm as mentioned previously, some specially algorithms are designed for the metric dimension and minimal doubly resolving set problem in hypercubes. For example, Nikolić et al. [28] designed a greedy algorithm and a dynamic programming procedure for the metric dimension of a hypercube by using the symmetry property of resolving sets to reduce the size of the feasible solution set. Hertz [14] designed an IP-based swapping algorithm for the metric dimension and minimal doubly resolving set problem in hypercubes.

The folded hypercube is a graph obtained by merging opposite vertices of a hypercube. A vertex of a folded $n$-cube $F_{n}$ is denoted by $[u]=\{u, \bar{u}\}$, where $u$ is a vertex of $Q_{n} . \quad[u][v] \in E\left(F_{n}\right)$ if and only if $u v \in E\left(Q_{n}\right)$ or $u \bar{v} \in E\left(Q_{n}\right)$ (see Figure 3). Note that $u v \in E\left(Q_{n}\right)$ if and only if $\bar{u} \in E\left(Q_{n}\right)$. It is easy to show that $d_{F_{n}}([u],[v])=\min \left\{d_{Q_{n}}(u, v), n-d_{Q_{n}}(u, v)\right\}$. Recently, Zhang et al. [33] gave the upper bound of metric dimension of folded $n$-cube.

Theorem 1.5 (Zhang et al. [33]). $\beta\left(F_{n}\right) \leq n-1$ for all odd $n \geq 5$ and $\beta\left(F_{n}\right) \leq 2 n-4$ for all even $n \geq 6$.

They raised the following conjecture.

Conjecture 1.1 (Zhang et al. [33]). If $n \geq 5$ is odd, then $\beta\left(F_{n}\right)=n-1$. 


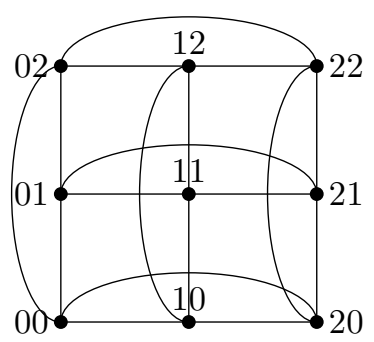

Figure 1. The graph $H_{2,3}$

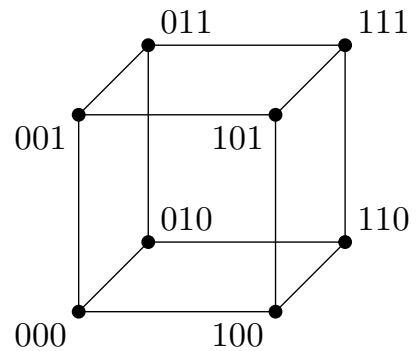

Figure 2. The graph $Q_{3}$

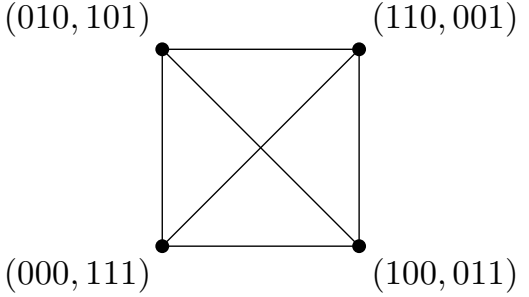

Figure 3. The graph $F_{3}$

The paper is organized as follows. Based on a new concept of doubly distance resolving sets, we reveal the relationship between resolving sets and doubly resolving sets in Section 2 . Using the preliminary results in Section 2, we show that $\Psi\left(H_{n, q}\right)=(2+o(1)) n / \log _{q} n$ and $\Psi\left(Q_{n}\right)=M(n)+1$ in Section 3 . Hence, we construct a bridge between the minimal doubly resolving set problem in hypercubes and the coin weighing problem. Using the result of the coin weighing problem, we prove that $\Psi\left(Q_{n}\right) \leq \Psi\left(Q_{n+1}\right) \leq \Psi\left(Q_{n}\right)+1$, which answer an open question in [14]. In Section 4, by exploring the connection of the metric dimension problems between hypercubes and folded hypercubes, we give a shorter proof for Theorem 1.5 and disprove Conjecture 1.1. Some asymptotic results of $\beta\left(F_{n}\right)$ and $\Psi\left(F_{n}\right)$ are also given. In Section 5 , we prove that the minimal doubly resolving set problem is NP-hard for split graphs, bipartite graphs and co-bipartite graphs. In Section 6, using the bridge between the minimal doubly resolving set problem in hypercubes and the coin weighing problem, we explore algorithms and experimental results for the minimal doubly resolving set problem in hypercubes and get some better upper bounds of $\Psi\left(Q_{n}\right)$ when $n \leq 93$. We also give precise values of $\beta\left(F_{n}\right)$ and $\Psi\left(F_{n}\right)$ when $n \leq 9$

\section{Preliminary results}

The following lemma is obvious but helpful to identify a doubly resolving set of a graph.

Lemma 2.1 (Kratica et al. [24]). Let $S=\left\{x_{1}, x_{2}, \ldots, x_{m}\right\}$ be a doubly resolving set of $G$. Then for every pair of distinct vertices $u, v \in V(G)$, there exists $x_{j} \in S$, such that $d_{G}\left(u, x_{1}\right)-d_{G}\left(u, x_{j}\right) \neq d_{G}\left(v, x_{1}\right)-d_{G}\left(v, x_{j}\right)$.

Now we introduce a new concept to reveal the relationship between resolving sets and doubly resolving sets of graphs. Let $G$ be a graph of order $n \geq 2$. Given a vertex $x \in V(G)$, a vertex subset $S$ of $G$ is a doubly distance resolving set of $G$ on $x$ if every pair of vertices $\{u, v\}$ with $d_{G}(u, x) \neq d_{G}(v, x)$ is doubly resolved by some pair of vertices in $S \cup\{x\}$. In other words, $S=\left\{x_{1}, x_{2}, \ldots, x_{m}\right\}$ is a doubly distance resolving set of $G$ on $x$ if and only if $d_{G}(u, x)$ is uniquely determined by the vector $\left(d_{G}(u, x)-d_{G}\left(u, x_{1}\right), d_{G}(u, x)-\right.$ $\left.d_{G}\left(u, x_{2}\right), \ldots, d_{G}(u, x)-d_{G}\left(u, x_{m}\right)\right)$ for any $u \in V(G)$.

Lemma 2.2. Let $S$ be a resolving set of $G$. Let $x \in S$ and $T$ be a doubly distance resolving set of $G$ on $x$. Then $S \cup T$ is a doubly resolving set of $G$.

Proof. Let $u, v$ be two distinct vertices of $G$. If $d_{G}(u, x) \neq d_{G}(v, x)$, then $\{u, v\}$ can be doubly resolved by the definition of $T$. If $d_{G}(u, x)=d_{G}(v, x)$, then there is a vertex $y$ such that $d_{G}(u, y) \neq d_{G}(v, y)$ by the definition of $S$ and we have $d_{G}(u, x)-d_{G}(u, y) \neq d_{G}(v, x)-d_{G}(v, y)$. It leads that $\{u, v\}$ is doubly resolved by $\{x, y\}$. Therefore, $S \cup T$ is a doubly resolving set of $G$.

Let $\phi(G, x)$ denote the minimum cardinality of a doubly distance resolving set of $G$ on $x$ and $\phi(G)=$ $\max \{\phi(G, x): x \in V(G)\}$.

Theorem 2.3. Let $G$ be a graph of order $n \geq 2$. Then

$$
\phi(G) \leq \Psi(G) \leq \beta(G)+\phi(G) .
$$


Proof. For every vertex $x$, since every doubly resolving set is a doubly distance resolving set on $x$ by definition, we have $\phi(G, x) \leq \Psi(G)$ and thus $\phi(G) \leq \Psi(G)$.

Let $S$ be a resolving set of $G$ with $|S|=\beta(G)$. Let $x \in S$ and $T$ be a doubly distance resolving set of $G$ on $x$ with $|T|=\phi(G, x)$. By Lemma $2.2, S \cup T$ is a doubly resolving set of $G$ and then $\Psi(G) \leq|S \cup T| \leq$ $|S|+|T|=\beta(G)+\phi(G, x) \leq \beta(G)+\phi(G)$.

A function $f: V(G) \rightarrow V(G)$ is an automorphism of $G$ if $f$ is bijective such that $u v \in E(G)$ if and only if $f(u) f(v) \in E(G)$. A graph $G$ is called a vertex-transitive graph if for every pair of vertices $\{x, y\}$, there is an automorphism $f$ such that $f(x)=y$.

Lemma 2.4. Let $G$ be a vertex-transitive graph of order $n \geq 2$. Then the following holds:

(a) $\phi(G)=\phi(G, x)$ for every $x \in G$.

(b) For every $x \in G$, there is a minimum (doubly) resolving set $S$ of $G$ such that $x \in S$.

Proof. We just prove (a). The proof of (b) is similar. Let $y$ be the vertex such that $\phi(G, y)=\phi(G)$. Let $S$ be a minimum doubly distance resolving set of $G$ on $x$. By the definition of vertex-transitive graph, there is an automorphism $f: V(G) \rightarrow V(G)$ such that $f(x)=y$. Then $f(S)=\{f(s): s \in S\}$ is a doubly distance resolving set of $G$ on $y$. It leads that $\phi(G)=\phi(G, y) \leq|f(S)|=\phi(G, x) \leq \phi(G)$, i.e. $\phi(G)=\phi(G, x)$.

\section{$3 \quad$ Hamming graphs and hypercubes}

Let $x, y$ be two vertices of $H_{n, q}$. It is clear that $f(u)=u-x+y$ is an automorphism of $H_{n, q}$ with $f(x)=y$. Then $H_{n, q}$ is a vertex-transitive graph. By the Lemma 2.4(b), we have the following corollary, which was proved for $q=2$ on the metric dimension problem in [28].

Corollary 3.1. There is a minimum (doubly) resolving set $S$ of $H_{n, q}$ such that $\overrightarrow{0} \in S$.

Lemma 3.2. For every positive integer $n, \phi\left(H_{n, q}\right) \leq \min \{q-1, n\}$.

Proof. Since $H_{n, q}$ is a vertex-transitive graph, it suffices to prove that $\phi\left(H_{n, q}, \overrightarrow{0}\right) \leq \min \{q-1, n\}$ by Lemma $2.4($ a)

Let $S=\{\overrightarrow{1}, \ldots, \overrightarrow{q-1}\}$. We will prove that $S$ is a doubly distance resolving set of $H_{n, q}$ on $\overrightarrow{0}$. Let $u$ be a vertex of $H_{n, q}$ and $f(u, c)=\sum_{i=1}^{n} 1_{u_{i}=c}$, where $1_{u_{i}=c}=1$ if $u_{i}=c$ and $1_{u_{i}=c}=0$ if $u_{i} \neq c$. Let $a_{u, c}=d_{H_{n, q}}(u, \overrightarrow{0})-d_{H_{n, q}}(u, \vec{c})$. Then we have

$$
a_{u, c}=\sum_{i=1}^{n} 1_{u_{i} \neq 0}-\sum_{i=1}^{n} 1_{u_{i} \neq c}=(n-f(u, 0))-(n-f(u, c))=f(u, c)-f(u, 0) .
$$

Since $\sum_{c=0}^{q-1} f(u, c)=n$, we have

$$
\sum_{c=1}^{q-1} a_{u, c}=\sum_{c=1}^{q-1} f(u, c)-(q-1) f(u, 0)=\sum_{c=1}^{q-1} f(u, c)-(q-1)\left(n-\sum_{c=1}^{q-1} f(u, c)\right)=q \sum_{c=1}^{q-1} f(u, c)-(q-1) n .
$$

Then

$$
d_{H_{n, q}}(u, \overrightarrow{0})=\sum_{c=1}^{q-1} f(u, c)=\frac{\sum_{c=1}^{q-1} a_{u, c}+(q-1) n}{q}
$$

Therefore, $d_{H_{n, q}}(u, \overrightarrow{0})$ is uniquely determined by the vector $\left(a_{u, 1}, \ldots, a_{u, q-1}\right)$, i.e. $S$ is a doubly distance resolving set of $H_{n, q}$ on $\overrightarrow{0}$.

Now we assume that $n \leq q-1$. Let $T=\{\overrightarrow{1}, \ldots, \vec{n}\}$. Then we will prove that $T$ is a doubly distance resolving set of $H_{n, q}$ on $\overrightarrow{0}$. If there is a pair of vertices $\{u, v\}$ such that $d_{H_{n, q}}(u, \overrightarrow{0}) \neq d_{H_{n, q}}(v, \overrightarrow{0})$ and $a_{u, c}=$ 
$a_{v, c}$ for all $c \in\{1, \ldots, n\}$, then, without loss of generality, we assume that $b=d_{H_{n, q}}(v, \overrightarrow{0})-d_{H_{n, q}}(u, \overrightarrow{0}) \geq 1$. We have $f(u, 0)=n-d_{H_{n, q}}(u, \overrightarrow{0})=n-\left(d_{H_{n, q}}(v, \overrightarrow{0})-b\right)=f(v, 0)+b$ and $f(u, c)=a_{u, c}+f(u, 0)=$ $a_{v, c}+f(v, 0)+b=f(v, c)+b$ for all $c \in\{1, \ldots, n\}$. But it leads that $\sum_{c=0}^{n} f(u, c)=\sum_{c=0}^{n}(f(v, c)+b) \geq n+1$, a contradiction.

By the Theorem 2.3, we have $\beta\left(H_{n, q}\right) \leq \Psi\left(H_{n, q}\right) \leq \beta\left(H_{n, q}\right)+\min \{q-1, n\}$. Then we immediately get the following theorem by Theorem 1.2.

Theorem 3.3. $\Psi\left(H_{n, q}\right)=(2+o(1)) n / \log _{q} n$ for all $q \geq 2$.

Now we focus on the special Hamming graph, that is the hypercube $Q_{n}$. We have

$$
d_{Q_{n}}(u, x)=\sum_{i=1}^{n}\left|u_{i}-x_{i}\right|=\sum_{i=1}^{n} u_{i}+x_{i}-2 u_{i} x_{i}=u \cdot \overrightarrow{1}+x \cdot \overrightarrow{1}-2(u \cdot x) .
$$

Note that $u \cdot x=\sum_{i=1}^{n} u_{i} x_{i}$ is the inner product of $u$ and $v$. Then

$$
d_{Q_{n}}(u, \overrightarrow{0})-d_{Q_{n}}(u, x)=2(u \cdot x)-x \cdot \overrightarrow{1} .
$$

Firstly, we prove the equivalence between the minimal doubly resolving set problem in hypercubes and the coin weighing problem.

Theorem 3.4. For every positive integer $n$, we have $\Psi\left(Q_{n}\right)=M(n)+1$.

Proof. We first prove that $M(n) \leq \Psi\left(Q_{n}\right)-1$. By Corollary 3.1, let $S$ be a doubly resolving set of $Q_{n}$ such that $\overrightarrow{0} \in S$ and $|S|=\Psi\left(Q_{n}\right)$. Now we need to prove that $S^{\prime}=S \backslash\{\overrightarrow{0}\}$ is a weighing strategy. Suppose not, then there are two distinct vertices $u, v \in V\left(Q_{n}\right)$, such that $u \cdot x=v \cdot x$ for each $x \in S^{\prime}$. It leads that $d_{Q_{n}}(u, \overrightarrow{0})-d_{Q_{n}}(u, x)=2(u \cdot x)-(x \cdot \overrightarrow{1})=2(v \cdot x)-(x \cdot \overrightarrow{1})=d_{Q_{n}}(v, \overrightarrow{0})-d_{Q_{n}}(v, x)$ for each $x \in S^{\prime}$. By Lemma 2.1, $S$ is not a doubly resolving set, a contradiction.

Now we prove that $\Psi\left(Q_{n}\right) \leq M(n)+1$. Let $S$ be a weighing strategy such that $|S|=M(n)$. Then we prove that $S^{\prime}=S \cup\{\overrightarrow{0}\}$ is a doubly resolving set. Suppose not, then there are two distinct vertices $u, v \in V\left(Q_{n}\right)$, such that $d_{Q_{n}}(u, \overrightarrow{0})-d_{Q_{n}}(u, x)=d_{Q_{n}}(v, \overrightarrow{0})-d_{Q_{n}}(v, x)$ for each $x \in S$. Then $u \cdot x=$ $\left(d_{Q_{n}}(u, \overrightarrow{0})-d_{Q_{n}}(u, x)+x \cdot \overrightarrow{1}\right) / 2=\left(d_{Q_{n}}(v, \overrightarrow{0})-d_{Q_{n}}(v, x)+x \cdot \overrightarrow{1}\right) / 2=v \cdot x$, a contradiction.

By Theorem 1.1, it is easy to know that

$$
\beta\left(Q_{n}\right) \leq \beta\left(Q_{n+1}\right)=\beta\left(Q_{n} \square K_{2}\right) \leq \beta\left(Q_{n}\right)+\Psi\left(K_{2}\right)-1=\beta\left(Q_{n}\right)+1 .
$$

But it is an open problem whether $\Psi\left(Q_{n}\right)$ has the similar property (see Hertz [14]). Since we know that the coin weighing problem and minimal doubly resolving set problem in hypercubes are equivalent, it is not difficult to answer this open problem using the result of the coin weighing problem.

Theorem 3.5. For every positive integer $n$, we have $\Psi\left(Q_{n}\right) \leq \Psi\left(Q_{n+1}\right) \leq \Psi\left(Q_{n}\right)+1$.

Proof. By Theorem 3.4, we need to prove that $M(n) \leq M(n+1) \leq M(n)+1$.

Let $u=\left(u_{1}, \ldots, u_{n}\right)$ and $v=\left(v_{1}, \ldots, v_{n}\right)$ be two distinct distribution of defective coins. Let $S$ be a weighing strategy for $n+1$ coins such that $|S|=M(n+1)$. Then there exists $x=\left(x_{1}, \ldots, x_{n+1}\right) \in S$, such that $u^{\prime} \cdot x \neq v^{\prime} \cdot x$, where $u^{\prime}=\left(u_{1}, \ldots, u_{n}, 0\right)$ and $v^{\prime}=\left(v_{1}, \ldots, v_{n}, 0\right)$. Let $x^{\prime}=\left(x_{1}, \ldots, x_{n}\right)$. Then $u \cdot x^{\prime}=u^{\prime} \cdot x \neq v^{\prime} \cdot x=v \cdot x^{\prime}$. Thus, $S^{\prime}=\left\{x^{\prime}=\left(x_{1}, \ldots, x_{n}\right): x=\left(x_{1}, \ldots, x_{n+1}\right) \in S\right\}$ is a weighing strategy for $n$ coins, i.e. $M(n) \leq M(n+1)$.

Let $u=\left(u_{1}, \ldots, u_{n+1}\right)$ and $v=\left(v_{1}, \ldots, v_{n+1}\right)$ be two distinct distribution of defective coins. If $u_{n+1} \neq$ $v_{n+1}$, then $u \cdot y \neq v \cdot y$ where $y=(0, \ldots, 0,1)$. Now we assume that $u_{n+1}=v_{n+1}$. Let $S$ be a weighing strategy for $n$ coins such that $|S|=M(n)$. Then there exists $x=\left(x_{1}, \ldots, x_{n}\right) \in S$, such that $u^{\prime} \cdot x \neq v^{\prime} \cdot x$, where $u^{\prime}=\left(u_{1}, \ldots, u_{n}\right)$ and $v^{\prime}=\left(v_{1}, \ldots, v_{n}\right)$. Let $x^{\prime}=\left(x_{1}, \ldots, x_{n}, 0\right)$. Then $u \cdot x^{\prime}=u^{\prime} \cdot x \neq v^{\prime} \cdot x=v \cdot x^{\prime}$. Thus, $S^{\prime}=y \cup\left\{x^{\prime}=\left(x_{1}, \ldots, x_{n}, 0\right): x=\left(x_{1}, \ldots, x_{n}\right) \in S\right\}$ is a weighing strategy for $n+1$ coins, i.e. $M(n+1) \leq M(n)+1$. 


\section{Folded hypercubes}

It is clear that $f([u])=[u-x+y]$ is an automorphism of $F_{n}$ with $f([x])=[x-x+y]=[y]$. Then $F_{n}$ is a vertex-transitive graph. In this section, we will use some precise values of $\beta\left(Q_{n}\right)$ and $\beta\left(F_{n}\right)$ that have calculated in $[2,5]$ (see Table 1 ).

\begin{tabular}{cccccccccc}
\hline$n$ & 1 & 2 & 3 & 4 & 5 & 6 & 7 & 8 & 9 \\
$\beta\left(Q_{n}\right)$ & 1 & 2 & 3 & 4 & 4 & 5 & 6 & 6 & 7 \\
$\beta\left(F_{n}\right)$ & - & 1 & 3 & 6 & 4 & 8 & 6 & 11 & - \\
\hline
\end{tabular}

Table 1. $\beta\left(Q_{n}\right)$ and $\beta\left(F_{n}\right), n \leq 9$

Lemma 4.1. For every integer $n \geq 3, \beta\left(F_{n}\right) \geq \beta\left(Q_{n}\right)$.

Proof. Let $f: V\left(F_{n}\right) \rightarrow V\left(Q_{n}\right)$ be a function such that $f([x])=x$ if $x_{1}=0$ and $f([x])=\bar{x}$ if $x_{1}=1$. If $S$ is a vertex set of $F_{n}$, then $f(S)=\{f([x]):[x] \in S\}$. It suffices to prove that if $S$ is a resolving set of $F_{n}$, then $f(S)$ is a resolving set of $Q_{n}$.

For every two distinct vertices $u, v \in V\left(Q_{n}\right)$ with $u \neq \bar{v}$, since $S$ is a resolving set of $F_{n}$, there is a vertex $[x] \in S$, such that $d_{F_{n}}([u],[x]) \neq d_{F_{n}}([v],[x])$ with $f([x])=x$. Let $d_{1}=d_{F_{n}}([u],[x])$ and $d_{2}=d_{F_{n}}([v],[x])$. Then $d_{Q_{n}}(u, x) \in\left\{d_{1}, n-d_{1}\right\}$ and $d_{Q_{n}}(v, x) \in\left\{d_{2}, n-d_{2}\right\}$. If $d_{Q_{n}}(u, x)=d_{Q_{n}}(v, x)$, since $d_{1} \neq d_{2}$, we have $d_{1}+d_{2}=n$. Since $\max \left\{d_{1}, d_{2}\right\} \leq n / 2$, we have $d_{1}=d_{2}=n / 2$, a contradiction. Therefore, $d_{Q_{n}}(u, x) \neq d_{Q_{n}}(v, x)$.

Now we consider the case that $u=\bar{v}$. Since $F_{n}$ is a vertex-transitive graph, we can assume that $u=\overrightarrow{0}, v=\overrightarrow{1}$. Note that for each $y \in V\left(Q_{n}\right)$, we have $d_{Q_{n}}(u, y)+d_{Q_{n}}(v, y)=n$. If there is a vertex $x \in f(S)$ such that $d_{Q_{n}}(u, x) \neq d_{Q_{n}}(v, x)$, then we have done. Otherwise, for each $x \in f(S)$, we have $d_{Q_{n}}(u, x)=$ $d_{Q_{n}}(v, x)=n / 2$. It leads that $n$ is even. Let $S^{\prime}=\left\{[x]: \sum_{i=1}^{n} x_{i}=n / 2\right\}$. Then $S \subseteq S^{\prime}$. However, $\{[s],[t]\}$ cannot be resolved by $S^{\prime}$, where $s=(1,0,0, \ldots, 0)$ and $t=(0,1,0, \ldots, 0)$, since $d_{F_{n}}([s],[x])=d_{F_{n}}([t],[x])=$ $n / 2-1$ for each $[x] \in S^{\prime}$. Then $S^{\prime}$ is not a resolving set of $F_{n}$, a contradiction.

What is more, we prove that equality holds if $n \geq 3$ is odd.

Lemma 4.2. If $n \geq 3$ is odd, then $\beta\left(F_{n}\right)=\beta\left(Q_{n}\right)$.

Proof. By Lemma 4.1, it suffices to prove that $\beta\left(F_{n}\right) \leq \beta\left(Q_{n}\right)$. Let $g: V\left(Q_{n}\right) \rightarrow V\left(F_{n}\right)$ be a function such that $g(x)=[x]$. If $S$ is a vertex set of $Q_{n}$, then $g(S)=\{[x]: x \in S\}$. It suffices to prove that if $S$ is a resolving set of $Q_{n}$, then $g(S)$ is a resolving set of $F_{n}$.

For every $x \in S$ and distinct vertices $[u],[v] \in V\left(F_{n}\right)$, if $[x]$ resolves $[u],[v]$, we have done. Otherwise, without loss of generality, we assume that $d_{Q_{n}}(u, x)=d_{F_{n}}([u],[x])=d_{F_{n}}([v],[x])=d_{Q_{n}}(v, x)$. Then $d_{Q_{n}}(u, x)-d_{Q_{n}}(v, x)=u \cdot \overrightarrow{1}-v \cdot \overrightarrow{1}-(2 u \cdot x-2 v \cdot x)=0$. It shows that $u \cdot \overrightarrow{1}+v \cdot \overrightarrow{1}$ is even.

Since $S$ is a resolving set of $Q_{n}$, there is a vertex $y \in S$ such that $d_{Q_{n}}(u, y) \neq d_{Q_{n}}(v, y)$. Let $d_{1}=d_{Q_{n}}(u, y)$ and $d_{2}=d_{Q_{n}}(v, y)$. Then $d_{F_{n}}([u],[y])=\min \left\{d_{1}, n-d_{1}\right\}$ and $d_{F_{n}}([v],[y])=\min \left\{d_{2}, n-d_{2}\right\}$. If $[y]$ does not resolve $[u]$ and $[v]$, then $d_{1}+d_{2}=n$. Besides, $d_{1}+d_{2}=2 y \cdot \overrightarrow{1}+u \cdot \overrightarrow{1}+v \cdot \overrightarrow{1}-(2 u \cdot y+2 v \cdot y)$. It leads that $u \cdot \overrightarrow{1}+v \cdot \overrightarrow{1}$ is odd, a contradiction.

If $n$ is even, equality does not hold in general, such as $\beta\left(F_{4}\right)=6 \neq 4=\beta\left(Q_{4}\right)$. If $n$ is even, the following lemma provides the upper bound.

Lemma 4.3. For every positive integer $n, \beta\left(F_{n+1}\right) \leq 2 \beta\left(Q_{n}\right)$.

Proof. For each $x \in V\left(Q_{n}\right)$, let $x^{0}=\left(x_{1}, \ldots, x_{n}, 0\right)$ and $x^{1}=\left(x_{1}, \ldots, x_{n}, 1\right)$ be the two vertices in $V\left(Q_{n+1}\right)$. It suffices to prove that if $S$ is a resolving set of $Q_{n}$, then $S^{\prime}=\left\{\left[x^{0}\right],\left[x^{1}\right]: x \in S\right\}$ is a resolving set of $F_{n+1}$.

Let $[u],[v] \in V\left(F_{n}\right)$ be two distinct vertices. Without loss of generality, we assume that $u_{n+1}=v_{n+1}=0$. Let $u^{\prime}=\left(u_{1}, \ldots, u_{n}\right)$ and $v^{\prime}=\left(v_{1}, \ldots, v_{n}\right)$. Then since $S$ is a resolving set of $Q_{n}$, there is a vertex $x \in S$ such that $d_{Q_{n}}\left(u^{\prime}, x\right) \neq d_{Q_{n}}\left(v^{\prime}, x\right)$. Let $d_{1}=d_{Q_{n}}\left(u^{\prime}, x\right)$ and $d_{2}=d_{Q_{n}}\left(v^{\prime}, x\right)$. Then $d_{F_{n+1}}\left([u],\left[x^{0}\right]\right)=\min \left\{d_{1}, n+\right.$ 
$\left.1-d_{1}\right\}, d_{F_{n+1}}\left([u],\left[x^{1}\right]\right)=\min \left\{d_{1}+1, n-d_{1}\right\}, d_{F_{n+1}}\left([v],\left[x^{0}\right]\right)=\min \left\{d_{2}, n+1-d_{2}\right\}, d_{F_{n+1}}\left([v],\left[x^{1}\right]\right)=$ $\min \left\{d_{2}+1, n-d_{2}\right\}$. If $\left[x^{0}\right]$ or $\left[x^{1}\right]$ resolves $\{[u],[v]\}$, we have done. Otherwise, we have $d_{1}+d_{2}=n+1$ and $\left(d_{1}+1\right)+\left(d_{2}+1\right)=n+1$, a contradiction.

Recall that $\beta\left(Q_{n+1}\right) \leq \beta\left(Q_{n}\right)+1$ and hence $\beta\left(Q_{n+m}\right) \leq \beta\left(Q_{n}\right)+m$. Since $\beta\left(Q_{5}\right)=4, \beta\left(Q_{n}\right) \leq$ $\beta\left(Q_{5}\right)+n-5=n-1$ if $n \geq 5$. By Lemma $4.2, \beta\left(F_{n}\right)=\beta\left(Q_{n}\right) \leq n-1$ for odd $n \geq 5$. By Lemma 4.3, $\beta\left(F_{n}\right) \leq 2 \beta\left(Q_{n-1}\right) \leq 2 n-4$ for even $n \geq 6$. This is a shorter proof of Theorem 1.5. Since $\beta\left(Q_{9}\right)=7$, $\beta\left(Q_{n}\right) \leq \beta\left(Q_{9}\right)+n-9=n-2$ for odd $n \geq 9$. It implies that Conjecture 1.1 is false. Furthermore, we have the following asymptotic result of $\beta\left(F_{n}\right)$ by Lemmas $4.1-4.3$ and $\beta\left(Q_{n}\right)=(2+o(1)) n / \log _{2} n$.

Theorem 4.4. If $n$ is odd, then $\beta\left(F_{n}\right)=(2+o(1)) n / \log _{2} n$. If $n$ is even, then $(2+o(1)) n / \log _{2} n \leq$ $\beta\left(F_{n}\right) \leq(4+o(1)) n / \log _{2} n$.

Now we consider the doubly distance resolving set of $F_{n}$.

Lemma 4.5. If $n=2 k+1 \geq 3$ is odd, then $\phi\left(F_{n}\right) \leq(n+1) / 2$.

Proof. Let $x^{i}=\left(x_{1}^{i}, \ldots, x_{n}^{i}\right) \in V\left(Q_{n}\right)$ such that

$$
x_{j}^{i}=\left\{\begin{array}{ll}
1 & j=2 i-1 \text { or } 2 i \\
0 & \text { otherwise }
\end{array} \text { for } 1 \leq i \leq k \quad \text { and } \quad x_{j}^{k+1}= \begin{cases}1 & j=2 k+1 \\
0 & \text { otherwise. }\end{cases}\right.
$$

Let $S=\left\{\left[x^{1}\right], \ldots,\left[x^{k+1}\right]\right\}$. Then it suffices to prove that $S$ is a doubly distance resolving set of $F_{n}$ on $[\overrightarrow{0}]$.

Let $[u]$ be a vertex of $F_{n}$. Without loss of generality, we assume that $d_{Q_{n}}(u, \overrightarrow{0}) \leq k$. Then $d_{F_{n}}([u],[\overrightarrow{0}])=$ $\sum_{j=1}^{n} u_{j}$. Let $a_{i}=d_{F_{n}}([u],[\overrightarrow{0}])-d_{F_{n}}\left([u],\left[x^{i}\right]\right)$. Then

$$
a_{i}=d_{Q_{n}}(u, \overrightarrow{0})-\min \left\{d_{Q_{n}}\left(u, x^{i}\right), d_{Q_{n}}\left(u, \overline{x^{i}}\right)\right\}=\max \left\{2 u \cdot x^{i}-x^{i} \cdot \overrightarrow{1}, 2 u \cdot \overline{x^{i}}-\overline{x^{i}} \cdot \overrightarrow{1}\right\} .
$$

Therefore

$$
a_{i}=\max \left\{2\left(u_{2 i-1}+u_{2 i}\right)-2,2\left(\sum_{j=1}^{n} u_{j}-u_{2 i}-u_{2 i-1}\right)-(n-2)\right\} \text { for } 1 \leq i \leq k
$$

and

$$
a_{k+1}=\max \left\{2 u_{2 k+1}-1,2\left(\sum_{j=1}^{n} u_{j}-u_{2 k+1}\right)-(n-1)\right\} .
$$

Firstly, if $a_{i}$ is even for every $i \leq k$ and $a_{k+1}$ is odd, then $a_{i}=2\left(u_{2 i-1}+u_{2 i}\right)-2$ for every $i \leq k$ and $a_{k+1}=2 u_{2 k+1}-1$. Thus,

$$
\sum_{j=1}^{n} u_{j}=\sum_{i=1}^{k+1} u \cdot x^{i}=\left(\sum_{i=1}^{k+1} a_{i}+n\right) / 2 .
$$

Secondly, if $a_{k+1}$ is even, then

$$
2\left(\sum_{j=1}^{n} u_{j}-u_{2 k+1}\right)-(n-1)>2 u_{2 k+1}-1 \Rightarrow 2 \sum_{j=1}^{n} u_{j}>4 u_{2 k+1}+n-2 \geq n-2=2 k-1 .
$$

Since $d_{Q_{n}}(u, \overrightarrow{0})=\sum_{j=1}^{n} u_{j} \leq k$, we have $\sum_{j=1}^{n} u_{j}=k$.

Finally, if $a_{i}$ is odd for some $i \leq k$, then $a_{i}=2\left(\sum_{j=1}^{n} u_{j}-u_{2 i}-u_{2 i-1}\right)-(n-2)$ and

$$
2\left(u_{2 i-1}+u_{2 i}\right)-2<2\left(\sum_{j=1}^{n} u_{j}-u_{2 i}-u_{2 i-1}\right)-(n-2) \Rightarrow u_{2 i-1}+u_{2 i}<\frac{2 \sum_{j=1}^{n} u_{j}-(n-4)}{4} \leq \frac{3}{4} .
$$


It leads that $u_{2 i-1}+u_{2 i}=0$. We have $\sum_{j=1}^{n} u_{j}=\left(a_{i}+n-2\right) / 2+\left(u_{2 i-1}+u_{2 i}\right)=\left(a_{i}+n-2\right) / 2$.

From the above discussion, $d_{F_{n}}([u],[\overrightarrow{0}])$ is uniquely determined by the vector $\left(a_{1}, \ldots, a_{k+1}\right)$. Therefore, $S$ is a doubly distance resolving set of $F_{n}$ on $[\overrightarrow{0}]$.

Lemma 4.6. If $n=2 k$ is even, then $\phi\left(F_{n}\right) \leq n-1$.

Proof. Let $x^{i}=\left(x_{1}^{i}, \ldots, x_{n}^{i}\right) \in V\left(Q_{n}\right)$ such that for $0 \leq i \leq n$,

$$
x_{j}^{i}= \begin{cases}1 & j \leq i \\ 0 & \text { otherwise }\end{cases}
$$

Let $S=\left\{\left[x^{1}\right], \ldots,\left[x^{n-1}\right]\right\}$. It suffices to prove that $S$ is a doubly distance resolving set of $F_{n}$ on $[\overrightarrow{0}]$.

Let $[u]$ be a vertex of $F_{n}$. Without loss of generality, we assume that $d_{Q_{n}}(u, \overrightarrow{0}) \leq k$. Then $d_{F_{n}}([u],[\overrightarrow{0}])=$ $\sum_{j=1}^{n} u_{j}$. Let $a_{i}=d_{F_{n}}([u],[\overrightarrow{0}])-d_{F_{n}}\left([u],\left[x^{i}\right]\right)$,

$$
b_{i}=d_{Q_{n}}(u, \overrightarrow{0})-d_{Q_{n}}\left(u, x^{i}\right)=2 u \cdot x^{i}-x^{i} \cdot \overrightarrow{1}=2 \sum_{j=1}^{i} u_{j}-i
$$

and

$$
c_{i}=d_{Q_{n}}(u, \overrightarrow{0})-d_{Q_{n}}\left(u, \overline{x^{i}}\right)=2 u \cdot \overline{x^{i}}-\overline{x^{i}} \cdot \overrightarrow{1}=2 \sum_{j=i+1}^{n} u_{j}-(n-i) .
$$

Then

$$
a_{i}=\max \left\{b_{i}, c_{i}\right\}=\max \left\{2 \sum_{j=1}^{i} u_{j}-i, 2 \sum_{j=i+1}^{n} u_{j}-(n-i)\right\} .
$$

Let $d_{i}=b_{i}-c_{i}$. First, since $b_{0}=c_{n}=0$, we have $d_{0} \leq 0$ and $d_{n} \geq 0$. Second, $\left|d_{i+1}-d_{i}\right| \leq\left|b_{i+1}-b_{i}\right|+$ $\left|c_{i+1}-c_{i}\right|=\left|2 u_{i+1}-1\right|+\left|1-2 u_{i+1}\right|=2$. Third, since $b_{i}+c_{i}=2 \sum_{j=1}^{n} u_{j}-n$ is even, $d_{i}$ is even. Combining them with the principle of bisection method, there is a $k$ such that $d_{k}=0$, i.e. $a_{k}=b_{k}=c_{k}$. Besides, for each $i$ such that $b_{i} \neq c_{i}$, we have $a_{i}=\max \left\{b_{i}, c_{i}\right\}>\left(b_{i}+c_{i}\right) / 2=\sum_{j=1}^{n} u_{j}-n / 2=\left(b_{k}+c_{k}\right) / 2=a_{k}$, i.e. $a_{k}=\min \left\{a_{i}: i \in\{0,1, \ldots, n\}\right\}$. Note that $a_{0}=a_{n}=0$. Therefore,

$$
\sum_{j=1}^{n} u_{j}=\sum_{j=1}^{k} u_{j}+\sum_{j=k+1}^{n} u_{j}=\frac{a_{k}+k}{2}+\frac{a_{k}+(n-k)}{2}=a_{k}+\frac{n}{2} .
$$

From the above, $d_{F_{n}}([u],[\overrightarrow{0}])$ is uniquely determined by the vector $\left(a_{1}, \ldots, a_{k+1}\right)$. Therefore, $S$ is a doubly distance resolving set of $F_{n}$ on $[\overrightarrow{0}]$.

By Theorems 2.3 and 4.4, we have the following theorem.

Theorem 4.7. If $n \geq 3$ is odd, then $\Psi\left(F_{n}\right) \leq \beta\left(F_{n}\right)+(n+1) / 2=n / 2+o(n)$. If $n$ is even, then $\Psi\left(F_{n}\right) \leq \beta\left(F_{n}\right)+n-1=n+o(n)$.

\section{$5 \quad$ NP-completeness}

A split graph is a graph whose vertex set is the disjoint union of a clique $C$ and an independent set $I$. In other words, every two vertices in $C$ are connected by an edge, while no two vertices in $I$ are connected by an edge. There is no restriction on edges having one end in $C$ and one end in $I$.

Similarly, a bipartite graph is a graph whose vertex set is the disjoint union of two independent sets $S_{1}$ and $S_{2}$. A co-bipartite graph is a graph whose vertex set is the disjoint union of two cliques $C_{1}$ and $C_{2}$. 
In this section, we prove that the minimal doubly resolving set problem is NP-hard even for split graphs, bipartite graphs and co-bipartite graphs. The proof is an extension of the proof for metric dimension problem in [10]. Besides, we omit some details that were mentioned in [10].

The 3-dimensional matching problem is defined as follows. Given three disjoint sets $A, B, C$ such that $|A|=|B|=|C|=n$, and a set of triples $S \subseteq A \times B \times C$, is there a subset $S^{\prime} \subseteq S$ such that each element of $A \cup B \cup C$ occurs in exactly one of the triples of $S^{\prime}$. It is well-known that 3-dimensional matching problem is NP-hard, due to Karp [18].

For each subset $S^{\prime} \subseteq S$, the cost of $S^{\prime}$ is calculated by $c\left(S^{\prime}\right)=\left|S^{\prime}\right|+3 n-\left|\bigcup_{(a, b, c) \in S^{\prime}}\{a, b, c\}\right|$. Note that if $S^{\prime}$ is a 3-dimensional matching, then $c\left(S^{\prime}\right)=n$. Let $N=2^{12} n$ and $n^{\prime}=n N$. Let $\mathcal{A}=\bigcup_{i=1}^{N} A_{i}, \mathcal{B}=\bigcup_{i=1}^{N} B_{i}$ and $\mathcal{C}=\bigcup_{i=1}^{N} C_{i}$, where $A_{i}, B_{i}, C_{i}$ are the copies of $A, B, C$ respectively. Let $\mathcal{S}=\bigcup_{i=1}^{N} S_{i}$, where $S_{i}$ is the copy of $S$ corresponding to $A_{i}, B_{i}, C_{i}$. It is clear that $\mathcal{S}^{\prime}=\bigcup_{i=1}^{N} S_{i}^{\prime} \subseteq \mathcal{S}$ is a 3-dimensional matching if $S^{\prime} \subseteq S$ is a 3 -dimensional matching and $S_{i}^{\prime}$ is the copy of $S^{\prime}$ corresponding to $A_{i}, B_{i}, C_{i}$. Furthermore, Epstein et al. [10] proved the following lemma.

Lemma 5.1 (Epstein et al. [10]). There is a 3-dimensional matching $S^{\prime} \subseteq S$ if and only if there is a subset $\mathcal{S}^{\prime} \subseteq \mathcal{S}$ such that $c\left(\mathcal{S}^{\prime}\right) \leq n^{\prime}+\sqrt{n^{\prime}}-1$.

Let $\mathcal{S}=\left\{s_{0}, s_{1}, \ldots, s_{\tau-1}\right\}, v=\left\lceil\log _{2} \tau\right\rceil$ and $K=n^{\prime}+v+5$. Note that $K<n^{\prime}+\sqrt{n^{\prime}}-4$. They construct a graph $G$ whose vertices are partitioned into two sets $I=\left\{s_{\mathcal{A}}, s_{\mathcal{B}}, s_{\mathcal{C}}, s_{\mathcal{D}}\right\} \cup \mathcal{S}$ and $J=\mathcal{A} \cup \mathcal{B} \cup$ $\mathcal{C} \cup\left\{d_{0}, d_{1}, \ldots, d_{v-1}\right\}$. If $u \in J$ and $v \in I$, then $\{u, v\} \in E$ in the seven following cases (see Figure 4):

1. $u \in \mathcal{A}$ and $v=s_{\mathcal{A}}$.

2. $u \in \mathcal{B}$ and $v=s_{\mathcal{B}}$.

3. $u \in \mathcal{C}$ and $v=s_{\mathcal{C}}$.

4. $u \in \mathcal{A} \cup \mathcal{B} \cup \mathcal{C}$ and $v=s_{\mathcal{D}}$.

5. $u \in\{a, b, c\}$ and $v=(a, b, c) \in \mathcal{S}$

6. $u=d_{i}$ and $v=s_{j}$ such that $\left\lfloor j / 2^{i}\right\rfloor \bmod 2=1$

7. $u=d_{i}$ and $v=s_{\mathcal{D}}$,

The set of additional edges of $G$ is defined according to the following cases. For the case of bipartite graphs there are no additional edges. For the case of split graphs, $J$ is a clique and $I$ is an independent set, and for the case of co-bipartite graphs both $I$ and $J$ are cliques. Clearly, the construction of the graph $G$ in all cases can be done in polynomial time. Then they prove the following lemma.

Lemma 5.2 (Epstein et al. [10]). (a) If $G$ has a resolving set $L$ such that $|L| \leq K$, then there is a subset $\mathcal{S}^{\prime} \subseteq \mathcal{S}$ such that $c\left(\mathcal{S}^{\prime}\right) \leq K+3<n^{\prime}+\sqrt{n^{\prime}}-1$.

(b) If there is a 3-dimensional matching $\mathcal{S}^{\prime} \subseteq \mathcal{S}$, then $L=\mathcal{S}^{\prime} \cup\left\{s_{\mathcal{A}}, s_{\mathcal{B}}, s_{\mathcal{C}}, s_{\mathcal{D}}\right\} \cup\left\{d_{0}, d_{1}, \ldots, d_{v-1}\right\}$ is a resolving set of $G$. Note that $|L|=K-1$.

Now let us consider the minimal doubly resolving set problem.

Lemma 5.3. (a) If $G$ has a doubly resolving set $L$ such that $|L| \leq K$, then there is a subset $\mathcal{S}^{\prime} \subseteq \mathcal{S}$ such that $c\left(\mathcal{S}^{\prime}\right) \leq K+3<n^{\prime}+\sqrt{n^{\prime}}-1$.

(b) If there is a 3-dimensional matching $\mathcal{S}^{\prime} \subseteq \mathcal{S}$, then $L=\mathcal{S}^{\prime} \cup\left\{s_{\mathcal{A}}, s_{\mathcal{B}}, s_{\mathcal{C}}, s_{\mathcal{D}}\right\} \cup\left\{d_{0}, d_{1}, \ldots, d_{v-1}\right\}$ is a doubly resolving set of $G$. Note that $|L|=K-1$.

Proof. By Lemma 5.2(a), we get (a) immediately since $L$ is also a resolving set. In order to prove (b), by Lemmas 2.2 and $5.2(\mathrm{~b})$, it suffices to prove that $L^{\prime}=\left\{s_{\mathcal{A}}, s_{\mathcal{B}}, s_{\mathcal{C}}\right\} \cup\left\{d_{0}, d_{1}, \ldots, d_{v-1}\right\}$ is a doubly distance resolving set on $s_{\mathcal{D}}$.

It is easy to check that for each $u \in V(G), d_{G}\left(u, s_{\mathcal{D}}\right) \leq 2$. In addition, for the case that $G$ is a co-bipartite graph, $d_{G}\left(u, s_{\mathcal{D}}\right) \leq 1$. Let $\{u, v\}$ be the pair of vertices with $d_{G}\left(u, s_{\mathcal{D}}\right) \neq d_{G}\left(v, s_{\mathcal{D}}\right)$. Then there exist only three possibilities:

Case 1: $u=s_{\mathcal{D}}$ and $d_{G}\left(v, s_{\mathcal{D}}\right)=1$. If $v \in\left\{d_{0}, d_{1}, \ldots, d_{v-1}\right\}$, then $\{u, v\}$ is doubly resolved by $\{u, v\}$. If $v \in \mathcal{A} \cup \mathcal{B} \cup \mathcal{C}$, then without loss of generality, we assume that $v \in \mathcal{A}$. Since $d_{G}(u, u)-d_{G}(v, u)=0-1<$ $1-1 \leq d_{G}\left(u, s_{\mathcal{A}}\right)-d_{G}\left(v, s_{\mathcal{A}}\right),\{u, v\}$ is doubly resolved by $\left\{u, s_{\mathcal{A}}\right\}$. The following situations only happen 


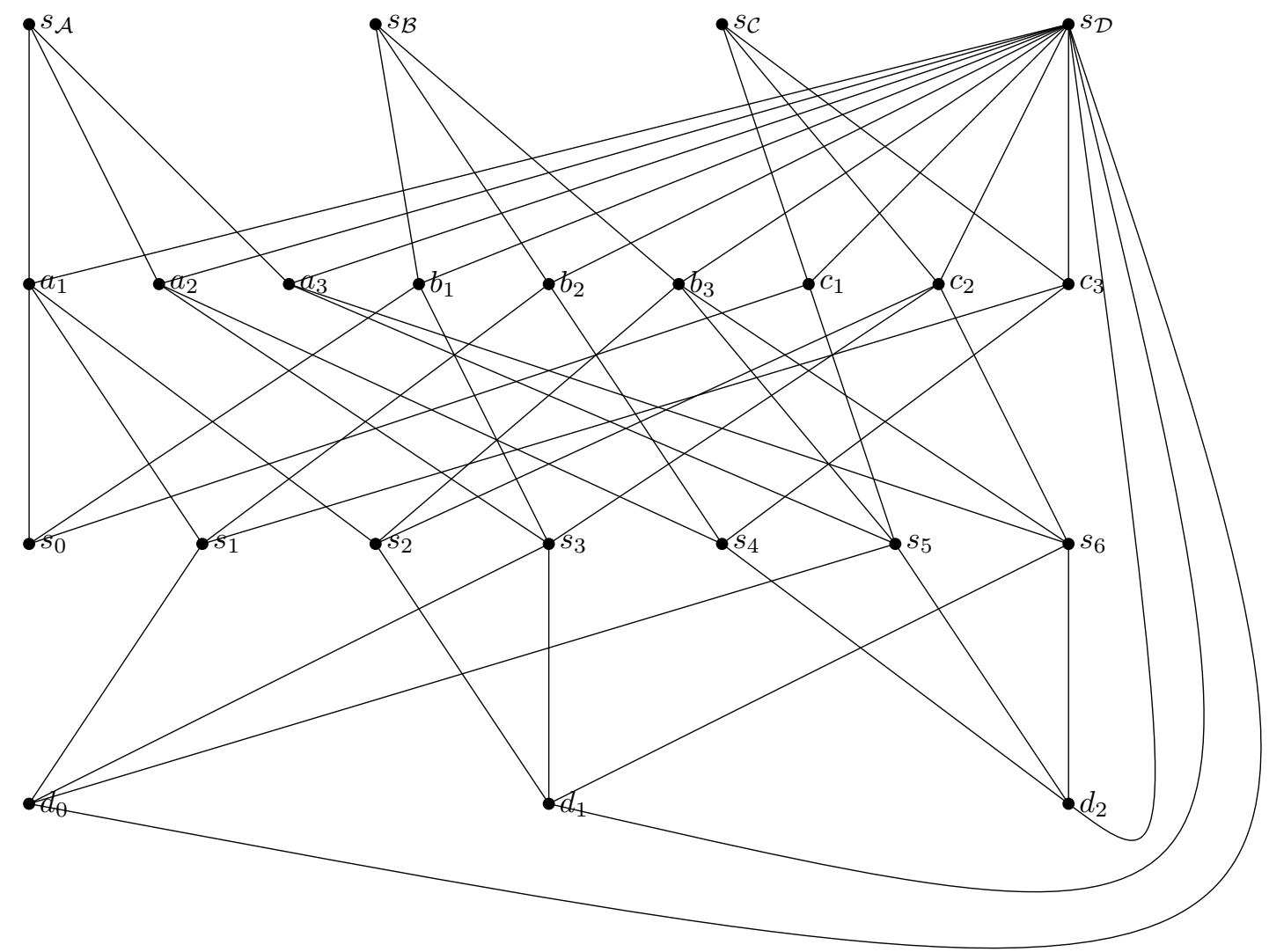

Figure 4. An example for constructing for the case of bipartite graphs (the instance actually is much larger), where $\mathcal{S}=\left\{\left(a_{1}, b_{1}, c_{1}\right),\left(a_{1}, b_{2}, c_{3}\right),\left(a_{1}, b_{3}, c_{2}\right),\left(a_{2}, b_{1}, c_{2}\right),\left(a_{2}, b_{2}, c_{3}\right),\left(a_{3}, b_{3}, c_{1}\right),\left(a_{3}, b_{3}, c_{2}\right)\right\}$

when $G$ is a co-bipartite graph. If $v \in\left\{s_{\mathcal{A}}, s_{\mathcal{B}}, s_{\mathcal{C}}\right\}$, then $\{u, v\}$ is doubly resolved by $\{u, v\}$. If $v \in S$, then $d_{G}(u, u)-d_{G}(v, u)=0-1<1-1=d_{G}\left(u, s_{\mathcal{A}}\right)-d_{G}\left(v, s_{\mathcal{A}}\right)$, i.e. $\{u, v\}$ is doubly resolved by $\left\{u, s_{\mathcal{A}}\right\}$.

Case 2: $u=s_{\mathcal{D}}$ and $d_{G}\left(v, s_{\mathcal{D}}\right)=2$. If $v \in\left\{s_{\mathcal{A}}, s_{\mathcal{B}}, s_{\mathcal{C}}\right\}$, then $\{u, v\}$ is doubly resolved by $\{u, v\}$. If $v \in S$, then $d_{G}(u, u)-d_{G}(v, u)=0-2<2-2=d_{G}\left(u, s_{\mathcal{A}}\right)-d_{G}\left(v, s_{\mathcal{A}}\right)$, i.e. $\{u, v\}$ is doubly resolved by $\left\{u, s_{\mathcal{A}}\right\}$.

Case 3: $d_{G}\left(u, s_{\mathcal{D}}\right)=1$ and $d_{G}\left(v, s_{\mathcal{D}}\right)=2$. If $u \in \mathcal{A} \cup \mathcal{B} \cup \mathcal{C}$, then without loss of generality, we assume that $u \in \mathcal{A}$. If $v \in S$, then $d_{G}\left(u, s_{\mathcal{D}}\right)-d_{G}\left(v, s_{\mathcal{D}}\right)=1-2<2-2 \leq d_{G}\left(u, s_{\mathcal{B}}\right)-d_{G}\left(v, s_{\mathcal{B}}\right)$, i.e. $\{u, v\}$ is doubly resolved by $\left\{s_{\mathcal{D}}, s_{\mathcal{B}}\right\}$. If $v \in\left\{s_{\mathcal{A}}, s_{\mathcal{B}}, s_{\mathcal{C}}\right\}$, then $d_{G}\left(u, s_{\mathcal{D}}\right)-d_{G}\left(v, s_{\mathcal{D}}\right)=1-2<1-0 \leq d_{G}(u, v)-d_{G}(v, v)$, i.e. $\{u, v\}$ is doubly resolved by $\left\{s_{\mathcal{D}}, v\right\}$. Now we assume that $u \in\left\{d_{0}, d_{1}, \ldots, d_{v-1}\right\}$. If $v \in S$, then $d_{G}\left(u, s_{\mathcal{D}}\right)-d_{G}\left(v, s_{\mathcal{D}}\right)=1-2<2-2 \leq d_{G}\left(u, s_{\mathcal{A}}\right)-d_{G}\left(v, s_{\mathcal{A}}\right)$, i.e. $\{u, v\}$ is doubly resolved by $\left\{s_{\mathcal{D}}, s_{\mathcal{A}}\right\}$. If $v \in\left\{s_{\mathcal{A}}, s_{\mathcal{B}}, s_{\mathcal{C}}\right\}$, then $d_{G}\left(u, s_{\mathcal{D}}\right)-d_{G}\left(v, s_{\mathcal{D}}\right)=1-2<2-0 \leq d_{G}(u, v)-d_{G}(v, v)$, i.e. $\{u, v\}$ is doubly resolved by $\left\{s_{\mathcal{D}}, v\right\}$.

Note that by Lemmas 5.1 and 5.3, there is a 3-dimensional matching $S^{\prime} \subseteq S$ if and only if $G$ has a doubly resolving set $L$ such that $|L| \leq K$. From the above, we get the following theorem.

Theorem 5.4. Given a value $K$ and a graph $G$ that is a split graph, a bipartite graph or a co-bipartite graph, deciding whether $\Psi(G) \leq K$ is NP-complete. 


\section{Algorithms and experimental results}

Lindström's construction of weighing strategy is a very creative method. For the details and the correctness, the interested reader is referred to Section 2.4 in [1].

Let $T$ be a finite set and $\mathcal{F} \subseteq 2^{T}$ a collection of subsets. $\mathcal{F}$ is called a (simplicial) complex if $A \in \mathcal{F}$ and $B \subseteq A$ imply $B \in \mathcal{F}$. Recall that $M(n)$ is the minimum number of weighings for $n$ coins. Lindström proved the following theorem.

Theorem 6.1 (Lindström $[26])$. $M\left(\sum_{A \in \mathcal{F}}|A|\right) \leq|\mathcal{F}|-1$ for every complex $\mathcal{F}$.

For a positive integer $m$, the binary representation can be written to $m=\sum_{i=1}^{t} 2^{k_{i}}$. Then let $F_{m}=$ $\left\{k_{1}, k_{2}, \ldots, k_{t}\right\}$ with $F_{0}=\emptyset$. For example, $10=(1010)_{2}=2^{1}+2^{3}$ and $F_{10}=\{1,3\}$. It is easy to know that $\mathcal{F}_{m}=\left\{F_{0}, F_{1}, \ldots, F_{m-1}\right\}$ is a complex. Based on Theorems 3.4 and 3.5, we can construct a doubly resolving set of $Q_{n}$ with cardinality $|\mathcal{F}|$ for every complex $\mathcal{F}$ and $n \leq \sum_{A \in \mathcal{F}}|A|$. Our algorithm for finding upper bounds of $\Psi\left(Q_{n}\right)$ is given in Algorithm 1 .

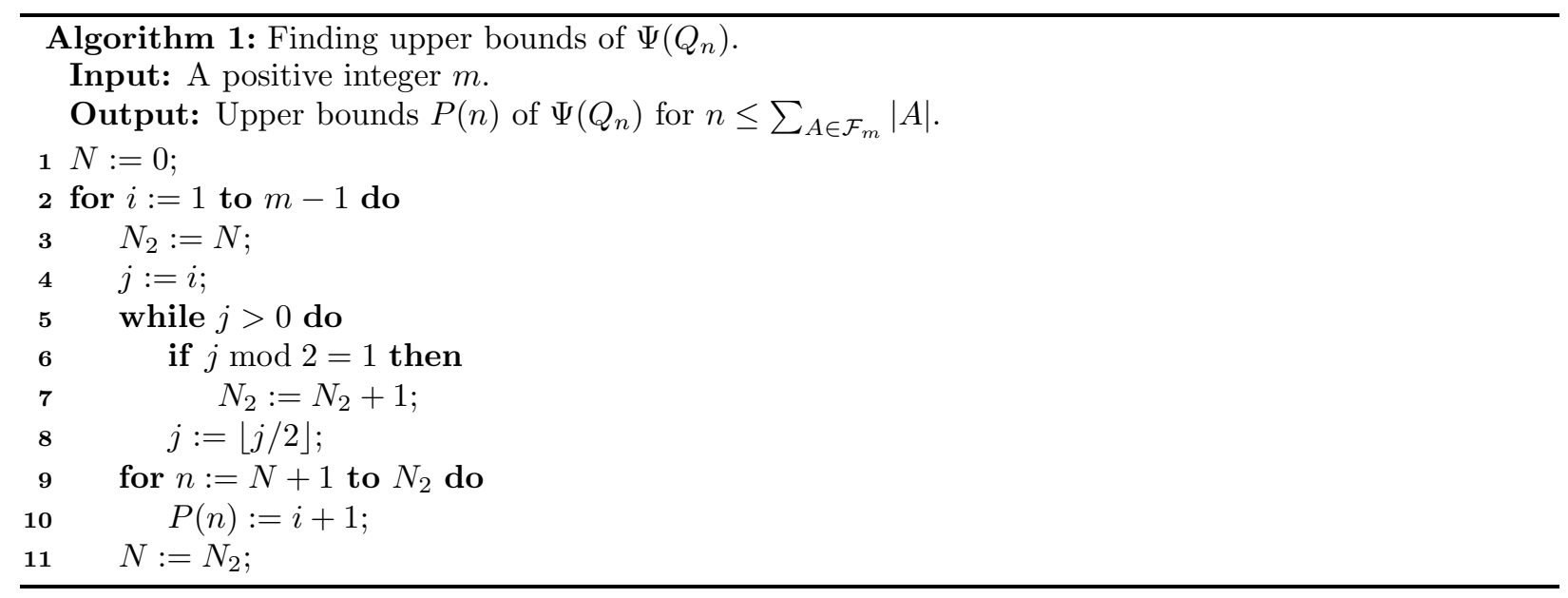

We use $\bar{\beta}_{n}$ and $\bar{\Psi}_{n}$ to denote the upper bounds of $\beta\left(Q_{n}\right)$ and $\Psi\left(Q_{n}\right)$, respectively. The genetic algorithm (GA), variable neighborhood search (VNS) algorithm and IP-based swapping (IPBS) algorithm were reported in [24], [27] and [14], respectively. Our computing method is Algorithm 1. Due to the limitation of the memory space and computing time, the previous results only compute for $n \leq 22$. Except for $n=14,16,18$, our upper bounds are same with them (see Table 2). Note that conversely their results actually improved the Lindström's upper bounds for coin weighing problem in $n=14,16,18$, i.e. $M(14) \leq 8$, $M(16) \leq 9$ and $M(18) \leq 10$. In addition, our upper bound of $\Psi\left(Q_{28}\right)$ is better than the upper bound of $\beta\left(Q_{28}\right)$ that founded by IPBS (see Table 3 ). Recall that $\beta\left(Q_{n}\right) \leq \Psi\left(Q_{n}\right)$. What is more, when $29 \leq n \leq 90$, all of our upper bounds of $\Psi\left(Q_{n}\right)$ are not more than the upper bounds of $\beta\left(Q_{n}\right)$ that is calculated by a dynamic programming (DP) procedure in [28] (see Table 4). Besides, some of our upper bounds of $\Psi\left(Q_{n}\right)$ are even better than their upper bounds of $\beta\left(Q_{n}\right)$. 


\begin{tabular}{ccccc}
\hline$n$ & GA & VNS & IPBS & Our \\
\hline 1 & 2 & - & - & 2 \\
2 & 3 & - & - & 3 \\
3 & 4 & - & - & 4 \\
4 & 4 & - & - & 4 \\
5 & 5 & - & - & 5 \\
6 & 6 & - & - & 6 \\
7 & 6 & - & - & 6 \\
8 & 7 & 7 & 7 & 7 \\
9 & 7 & 7 & 7 & 7 \\
10 & 8 & 8 & 8 & 8 \\
11 & 8 & 8 & 8 & 8 \\
12 & 9 & 8 & 8 & 8 \\
13 & 9 & 9 & 9 & 9 \\
14 & 10 & 9 & 9 & 10 \\
15 & 10 & 10 & 10 & 10 \\
16 & 11 & 10 & 10 & 11 \\
17 & 12 & 11 & 11 & 11 \\
18 & - & - & 11 & 12 \\
19 & - & - & 12 & 12 \\
20 & - & - & 12 & 12 \\
21 & - & - & 13 & 13 \\
22 & - & - & 13 & 13 \\
\hline
\end{tabular}

Table 2. $\bar{\Psi}_{n}, n \leq 22$

\begin{tabular}{ccc}
\hline$n$ & $\bar{\beta}_{n}$ (IPBS) & $\bar{\Psi}_{n}$ (Our) \\
\hline 23 & 13 & 14 \\
24 & 14 & 14 \\
25 & 14 & 14 \\
26 & 15 & 15 \\
27 & 15 & 15 \\
28 & 16 & 15 \\
\hline
\end{tabular}

Table 3. $\bar{\beta}_{n}$ and $\bar{\Psi}_{n}, 23 \leq n \leq 28$

\begin{tabular}{cccccccccccccc}
\hline$n$ & 29 & 30 & 31 & 32 & 33 & 34 & 35 & 36 & 37 & 38 & 39 & 40 & 41 \\
$\bar{\beta}_{n}(\mathrm{DP})$ & 16 & 16 & 16 & 16 & 17 & 18 & 19 & 19 & 20 & 21 & 21 & 22 & 22 \\
$\bar{\Psi}_{n}($ Our $)$ & 16 & 16 & 16 & 16 & 17 & 18 & 18 & 19 & 19 & 20 & 20 & 20 & 21 \\
\hline$n$ & 42 & 43 & 44 & 45 & 46 & 47 & 48 & 49 & 50 & 51 & 52 & 53 & 54 \\
$\bar{\beta}_{n}(\mathrm{DP})$ & 23 & 23 & 23 & 24 & 24 & 25 & 25 & 26 & 27 & 28 & 28 & 29 & 30 \\
$\bar{\Psi}_{n}(\mathrm{Our})$ & 21 & 22 & 22 & 22 & 23 & 23 & 23 & 24 & 24 & 24 & 24 & 25 & 25 \\
\hline$n$ & 55 & 56 & 57 & 58 & 59 & 60 & 61 & 62 & 63 & 64 & 65 & 66 & 67 \\
$\bar{\beta}_{n}(\mathrm{DP})$ & 30 & 30 & 31 & 31 & 32 & 32 & 32 & 32 & 32 & 32 & 32 & 32 & 32 \\
$\bar{\Psi}_{n}(\mathrm{Our})$ & 26 & 26 & 26 & 27 & 27 & 27 & 28 & 28 & 28 & 28 & 29 & 29 & 29 \\
\hline$n$ & 68 & 69 & 70 & 71 & 72 & 73 & 74 & 75 & 76 & 77 & 78 & 79 & 80 \\
$\bar{\beta}_{n}(\mathrm{DP})$ & 32 & 32 & 32 & 32 & 32 & 32 & 32 & 32 & 32 & 32 & 32 & 32 & 32 \\
$\bar{\Psi}_{n}(\mathrm{Our})$ & 30 & 30 & 30 & 30 & 31 & 31 & 31 & 31 & 32 & 32 & 32 & 32 & 32 \\
\hline$n$ & 81 & 82 & 83 & 84 & 85 & 86 & 87 & 88 & 89 & 90 & 91 & 92 & 93 \\
$\bar{\beta}_{n}(\mathrm{DP})$ & 33 & 34 & 35 & 35 & 36 & 37 & 37 & 38 & 38 & 39 & - & - & - \\
$\bar{\Psi}_{n}(\mathrm{Our})$ & 33 & 34 & 34 & 35 & 35 & 36 & 36 & 36 & 37 & 37 & 38 & 38 & 38 \\
\hline
\end{tabular}

Table $4 . \bar{\beta}_{n}$ and $\bar{\Psi}_{n}, 29 \leq n \leq 93$

Recall that Chartrand et al. [7] and Kratica et al. [24] have given the 0-1 integer linear programming formulations for the metric dimension problem and the minimal doubly resolving set problem respectively. Using the similar method, we give the $0-1$ integer linear programming formulations for computing $\phi(G, s)$.

For a doubly distance resolving set $S$ of $G$ on $s$, let

$$
x_{t}= \begin{cases}1 & \text { if } t \in S \\ 0 & \text { otherwise. }\end{cases}
$$


Let $T(G, s)=\left\{(u, v): d_{G}(u, s) \neq d_{G}(v, s)\right\}$. Let

$$
A_{(u, v),(s, t)}= \begin{cases}1 & \text { if } d_{G}(u, s)-d_{G}(u, t) \neq d_{G}(v, s)-d_{G}(v, t) \\ 0 & \text { otherwise. }\end{cases}
$$

The following 0-1 linear programming model of calculating the value of $\phi(G, s)$ can be formulated as:

$$
\begin{array}{lll}
\min & \sum_{t \in V(G)} x_{t} & \\
\text { s.t. } & \sum_{t \in V(G)} A_{(u, v),(s, t)} x_{t} \geq 1 & \forall(u, v) \in T(G, s) \\
& x_{t} \in\{0,1\} & \forall t \in V(G) .
\end{array}
$$

By Lemma 2.1, it is easy to see that each feasible solution of (2)-(3) defines a doubly distance resolving set $S$ of $G$ on $s$, and vice versa.

We use $0-1$ linear programming model to compute $\beta\left(F_{n}\right), \phi\left(F_{n}\right)$ and $\Psi\left(F_{n}\right)$ for $n \leq 10$ by Gurobi Optimizer (see Table 5). Note that the values of $\beta\left(F_{n}\right)$ for $n \leq 8$ have computed in [2].

\begin{tabular}{cccccccccc}
\hline$n$ & 2 & 3 & 4 & 5 & 6 & 7 & 8 & 9 & 10 \\
$\beta\left(F_{n}\right)$ & 1 & 3 & 6 & 4 & 8 & 6 & 11 & 7 & $\leq 14$ \\
$\Psi\left(F_{n}\right)$ & 2 & 3 & 6 & 5 & 9 & 6 & 11 & 7 & $\leq 14$ \\
$\phi\left(F_{n}\right)$ & 1 & 1 & 3 & 2 & 5 & 3 & 6 & 3 & $\leq 8$ \\
\hline
\end{tabular}

Table 5. $\beta\left(F_{n}\right), \phi\left(F_{n}\right)$ and $\Psi\left(F_{n}\right), n \leq 10$

\section{Open problems}

In Section 3, we proved that $\Psi\left(H_{n, q}\right) \leq \beta\left(H_{n, q}\right)+q-1$. However, we do not know whether it is best possible. We pose the following question.

Question 7.1. For every positive integer n, determine the smallest positive value $f(q)$, such that $\Psi\left(H_{n, q}\right) \leq$ $\beta\left(H_{n, q}\right)+f(q)$.

By the values of $\beta\left(F_{n}\right)$ and $\Psi\left(F_{n}\right)$ for $n \leq 10$ in Table 5 , we raise the following conjecture.

Conjecture 7.1. For every integer $n \geq 2, \beta\left(F_{n}\right) \leq \Psi\left(F_{n}\right) \leq \beta\left(F_{n}\right)+1$.

We observe that $\beta\left(F_{2 n}\right) \approx 2 \beta\left(F_{2 n-1}\right), \Psi\left(F_{2 n}\right) \approx 2 \Psi\left(F_{2 n-1}\right)$ and $\phi\left(F_{2 n}\right) \approx 2 \phi\left(F_{2 n-1}\right)$ when $n$ is small by Table 5. Besides, it seems to remain true when $n$ is large by comparing Lemma 4.2 with Lemma 4.3 , as well as comparing Lemma 4.5 with Lemma 4.6. We pose the following question and conjecture that the values are 2 .

Question 7.2. Determine the values of

$$
\lim _{n \rightarrow+\infty} \frac{\beta\left(F_{2 n}\right)}{\beta\left(F_{2 n-1}\right)}, \lim _{n \rightarrow+\infty} \frac{\Psi\left(F_{2 n}\right)}{\Psi\left(F_{2 n-1}\right)}, \lim _{n \rightarrow+\infty} \frac{\phi\left(F_{2 n}\right)}{\phi\left(F_{2 n-1}\right)} .
$$

\section{References}

[1] M. Aigner, Combinatorial search, Wiley-Teubner Series in Computer Science, John Wiley \& Sons, Ltd., Chichester; B. G. Teubner, Stuttgart, 1988. 
[2] R. F. Bailey, The metric dimension of small distance-regular and strongly regular graphs, Australas. J. Combin. 62 (2015) 18-34.

[3] R. F. Bailey, P. J. Cameron, Base size, metric dimension and other invariants of groups and graphs, Bull. Lond. Math. Soc. 43 (2) (2011) 209-242.

[4] Z. Beerliova, F. Eberhard, T. Erlebach, A. Hall, M. Hoffmann, M. Mihal'ák, L. S. Ram, Network discovery and verification, in: Graph-theoretic concepts in computer science, vol. 3787 of Lecture Notes in Comput. Sci., Springer, Berlin, 127-138, 2005.

[5] J. Cáceres, C. Hernando, M. Mora, I. M. Pelayo, M. L. Puertas, C. Seara, D. R. Wood, On the metric dimension of Cartesian products of graphs, SIAM J. Discrete Math. 21 (2) (2007) 423-441.

[6] D. G. Cantor, W. H. Mills, Determination of a subset from certain combinatorial properties, Canadian J. Math. 18 (1966) 42-48.

[7] G. Chartrand, L. Eroh, M. A. Johnson, O. R. Oellermann, Resolvability in graphs and the metric dimension of a graph, Discrete Appl. Math. 105 (1-3) (2000) 99-113.

[8] X. Chen, C. Wang, Approximability of the minimum weighted doubly resolving set problem, in: Computing and combinatorics, vol. 8591 of Lecture Notes in Comput. Sci., Springer, Cham, 357-368, 2014.

[9] V. Chvátal, Mastermind, Combinatorica 3 (3-4) (1983) 325-329.

[10] L. Epstein, A. Levin, G. J. Woeginger, The (weighted) metric dimension of graphs: hard and easy cases, Algorithmica 72 (4) (2015) 1130-1171.

[11] P. Erdős, A. Rényi, On two problems of information theory, Magyar Tud. Akad. Mat. Kutató Int. Közl. 8 (1963) 229-243.

[12] F. Harary, R. A. Melter, On the metric dimension of a graph, Ars Combin. 2 (1976) 191-195.

[13] C. Hernando, M. Mora, I. M. Pelayo, C. Seara, D. R. Wood, Extremal graph theory for metric dimension and diameter, Electron. J. Combin. 17 (1) (2010) Research Paper 30, 28.

[14] A. Hertz, An IP-based swapping algorithm for the metric dimension and minimal doubly resolving set problems in hypercubes, Optim. Lett. 14 (2) (2020) 355-367.

[15] Z. Jiang, N. Polyanskii, On the metric dimension of Cartesian powers of a graph, J. Combin. Theory Ser. A 165 (2019) 1-14.

[16] G. Kabatianski, V. Lebedev, J. Thorpe, The Mastermind game and the rigidity of the Hamming space, in: 2000 IEEE International Symposium on Information Theory (Cat. No. 00CH37060), IEEE, 375, 2000 .

[17] G. A. Kabatyanskiŭ, V. S. Lebedev, On the metric dimension of nonbinary Hamming spaces, Problemy Peredachi Informatsii 54 (1) (2018) 54-62.

[18] R. M. Karp, Reducibility among combinatorial problems, in: Complexity of computer computations (Proc. Sympos., IBM Thomas J. Watson Res. Center, Yorktown Heights, N.Y., 1972), 85-103, 1972.

[19] S. Khuller, B. Raghavachari, A. Rosenfeld, Landmarks in graphs, Discrete Appl. Math. 70 (3) (1996) 217-229.

[20] D. E. Knuth, The computer as master mind, J. Recreational Math. 9 (1) (1976/77) 1-6.

[21] J. Kratica, V. Kovačević-Vujčić, M. Čangalović, Computing the metric dimension of graphs by genetic algorithms, Comput. Optim. Appl. 44 (2) (2009) 343-361. 
[22] J. Kratica, V. Kovačević-Vujčić, M. Čangalović, M. Stojanović, Minimal doubly resolving sets and the strong metric dimension of Hamming graphs, Appl. Anal. Discrete Math. 6 (1) (2012) 63-71.

[23] J. Kratica, V. Kovačević-Vujčić, M. Čangalović, M. Stojanović, Minimal doubly resolving sets and the strong metric dimension of some convex polytopes, Appl. Math. Comput. 218 (19) (2012) 9790-9801.

[24] J. Kratica, M. Čangalović, V. Kovačević-Vujčić, Computing minimal doubly resolving sets of graphs, Comput. Oper. Res. 36 (7) (2009) 2149-2159.

[25] B. Lindström, On a combinatory detection problem. I, Magyar Tud. Akad. Mat. Kutató Int. Közl. 9 (1964) 195-207.

[26] B. Lindström, On a combinatorial problem in number theory, Canad. Math. Bull. 8 (1965) 477-490.

[27] N. Mladenović, J. Kratica, V. Kovačević-Vujčić, M. Čangalović, Variable neighborhood search for metric dimension and minimal doubly resolving set problems, European J. Oper. Res. 220 (2) (2012) 328-337.

[28] N. Nikolić, M. Čangalović, I. Grujičić, Symmetry properties of resolving sets and metric bases in hypercubes, Optim. Lett. 11 (6) (2017) 1057-1067.

[29] A. Sebő, E. Tannier, On metric generators of graphs, Math. Oper. Res. 29 (2) (2004) 383-393.

[30] H. S. Shapiro, Problem E1399, Amer. Math. Monthly 67 (1) (1960) 82.

[31] H. S. Shapiro, N. J. Fine, Elementary Problems and Solutions: Solutions: E1399, Amer. Math. Monthly 67 (7) (1960) 697-698.

[32] P. J. Slater, Leaves of trees, in: Proceedings of the Sixth Southeastern Conference on Combinatorics, Graph Theory, and Computing (Florida Atlantic Univ., Boca Raton, Fla., 1975), 549-559. Congressus Numerantium, No. XIV, 1975.

[33] Y. Zhang, L. Hou, B. Hou, W. Wu, D.-Z. Du, S. Gao, On the metric dimension of the folded $n$-cube, Optim. Lett. 14 (1) (2020) 249-257. 